\title{
Collagen triple helix repeat containing 1 promotes tumor angiogenesis in gastrointestinal stromal tumors
}

\author{
SENG WANG FU*, HAI YING CHEN* ${ }^{*}$ XIAO LU LIN, LI YANG and ZHI ZHENG GE \\ Division of Gastroenterology and Hepatology, Shanghai Institute of Digestive Disease, \\ Key Laboratory of Gastroenterology and Hepatology, Ministry of Health, Renji Hospital, \\ School of Medicine, Shanghai Jiao-Tong University, Shanghai 200001, P.R. China
}

Received November 15, 2015; Accepted November 25, 2016

DOI: $10.3892 / \mathrm{ol} .2017 .7111$

\begin{abstract}
Collagen triple helix repeat containing 1 (Cthrc1) is a secreted protein that has been observed to lead to poorer prognosis by inducing the invasion and metastasis in different tumors; however, it has not been demonstrated that Cthrc1 is involved in tumor angiogenesis. Immunohistochemical staining of Cthrc1 and CD31 in gastrointestinal stromal tumor tissue demonstrated that Cthrcl is associated with microvascular density. Overexpression of Cthrc1 protein may alter the properties of human umbilical vein endothelial cells (HUVECs), including migration, invasion, tubule formation and aortic ring sprouting. Small interfering RNA-mediated knockdown of Cthrc1 was performed to verify the opposite effects. Migration and tubule formation induced by Cthrc1 overexpression in HUVECs was attenuated by inhibition of phosphorylation in extracellular-signal-regulated protein kinase (ERK) and c-Jun N-terminal kinase (JNK) signaling pathways. The pro-angiogenic effect of Cthcrl is associated with increased phosphorylation of ERK and JNK in HUVECs. Silencing the expression of Cthrc1 protein may be a promising strategy to inhibit tumor angiogenesis.
\end{abstract}

\section{Introduction}

Angiogenesis is a complex process that includes adhesion, migration, invasion, proliferation and differentiation in endothelial cells (1). Neovascularization is necessary for tumor

Correspondence to: Professor Zhi Zheng Ge, Division of Gastroenterology and Hepatology, Shanghai Institute of Digestive Disease, Key Laboratory of Gastroenterology and Hepatology, Ministry of Health, Renji Hospital, School of Medicine, Shanghai Jiao-Tong University, 145 Middle Shandong Road, Shanghai 200001, P.R. China

E-mail: zhizhengge@aliyun.com

${ }^{*}$ Contributed equally

Key words: collagen triple helix repeat containing 1, extracellular-signal-regulated kinases, c-Jun $\mathrm{N}$-terminal kinase, human umbilical vein endothelial cells, angiogenesis growth by providing oxygen anxd nutrients (2). The tumor and the surrounding microenvironment, including cancer cells, endothelial cells, fibroblasts and immune cells, are associated and interact constantly $(3,4)$. Tumors may influence the microenvironment by releasing extracellular signals, including vascular endothelial growth factor, tumor necrosis factor $\alpha$, fibroblast growth factor 2 and interleukin 6 into the extracellular matrix (ECM), and promoting tumor-associated angiogenesis $(5,6)$.

Collagen triple helix repeat containing 1 (Cthrc1) was identified to encode a secreted protein that serves a role in the cellular response to arterial injury through vascular remodeling (7). It was demonstrated that Cthrc1 is related to vascular remodeling by inhibiting collagen production and fibrogenesis, and by promoting cell migration (8). The majority of Cthrcl studies have focused on its effects and underlying molecular mechanism, in promoting tumor cell invasion and metastasis (9-14). In the present study, it was demonstrated that increased expression of Cthrcl protein in gastrointestinal stromal tumor (GIST) is associated with increased microvascular density (MVD) in a tissue microarray; however, to the best of our knowledge, no previous study has demonstrated the effect of Cthrc1 in endothelial angiogenesis.

\section{Materials and methods}

Immunohistochemical (IHC) staining and evaluation. Two continuous tissue microarrays (no. HDgS-GIST060CS-01) including surgical tissues from 60 GISTs were purchased from Shanghai Outdo Biotech Co., Ltd. (Shanghai, China). The 60 patients included 33 females and 27 males, with a median age of $53.4 \pm 13.70$ years. The location of the samples included 5 cases in the stomach, 21 cases in the small intestine, 7 cases in the colon, 3 cases in the peritoneum and 2 cases in the mesentery. Sections were dewaxed in dimethylbenzene, hydrated in ethanol, incubated with $3 \%$ oxydol to inactivate endogenous peroxidase and incubated with a citrate solution for $30 \mathrm{~min}$ at $95^{\circ} \mathrm{C}$ for antigen retrieval. Sections were blocked with goat serum for $30 \mathrm{~min}$ and incubated with Anti-Cthrc1 (dilution, 1:200; cat. no. AP8778a; Abgent, San Diego, CA, USA) or anti-CD31 (dilution, 1:200; 11265-1-AP; ProteinTech, Chicago, IL, USA) at $4^{\circ} \mathrm{C}$ overnight. Sections were then incubated with a horseradish peroxidase (HRP)-labeled 
goat anti-mouse/rabbit antibody (dilution, ready to use: cat. no. D-3004; Shanghai Long Island Biotec. Co., Ltd., Shanghai, China) at $30^{\circ} \mathrm{C}$ for $30 \mathrm{~min}$ and DAB for $30 \mathrm{sec}$ (Fuzhou Maixin Biotechnology Development Co., Ltd., Fuzhou, China) at room temperature, followed by hematoxylin staining and mounting. Images were captured using a microscope (CX31-LV320; Olympus Corporation, Tokyo, Japan). CD31 staining results were evaluated by the amount of vessels per x200 fields (Fig. 1A-C). Cthrc1 staining results were evaluated as follows: -, no tumor cells stained;,$+<25 \%$ tumor cells stained (Fig. 1D); ,$++ 25-50 \%$ tumor cells stained (Fig. 1E);,$+++>50 \%$ tumor cells stained (Fig. 1F).

Cell culture.Human umbilical vein endothelial cells (HUVECs) were purchased from China Center for Type Culture Collection (Beijing, China). HUVECs were cultured in Dulbecco's modified Eagle's medium (DMEM; Gibco; Thermo Fisher Scientific, Inc., Waltham, MA, USA) supplemented with $10 \%$ fetal bovine serum (FBS; ScienCell Research Laboratories, Inc., Carlsbad, CA, USA) at $37^{\circ} \mathrm{C}$ in a humidified atmosphere of $5 \% \mathrm{CO}_{2}$ for $72 \mathrm{~h}$ prior to use. U0126 (Selleck Chemicals, Houston, TX, USA) and SP600125 (Selleck Chemicals) were used to inhibit extracellular-signal-regulated kinase 1/2 (ERK1/2) and c-Jun N-terminal kinase (JNK) phosphorylation, respectively. U0126 and SP600125 were dissolved in Dimethyl sulfoxide (DMSO; Sigma-Aldrich; Merck Millipore, Darmstadt, Germany), so DMSO was used as a phosphorylation control. The final concentration of U0126 and SP600125 was $10 \mu \mathrm{M}$ in the culture medium for the experiments.

Cthrcl-expressing adenoviral vector. An adenoviral vector expressing the human Cthrcl coding sequence under the control of a mouse cytomegalovirus promoter was constructed as described previously (15). A control adenoviral vector expressing green fluorescent protein was prepared in parallel (15).

Small interfering RNA (siRNA) experiments. Cthrc1 was knocked down using siRNA (siCTHRC1) and the synthetic duplex oligomers 5'-CCCAUUGAAGCUAUAAUUU-3' and 5'-AAAUUAUAGCUUCAAUGGG-3', purchased from Shanghai GenePharma Co., Ltd. (Shanghai, China). HUVECs were transiently transfected with siRNAs using GenMute ${ }^{\mathrm{TM}}$ reagent (SignaGen Laboratories, Rockville, MD, USA), according to the manufacturer's protocol.

Aortic ring assay. A total of 6 male Sprague-Dawley rats (8-weeks old) weighing 320-345 g were purchased from Shanghai SLAC Laboratory Animal Co., Ltd. The mice were sacrificed on the day of purchase. Chloral hydrate $(10 \%)$ was injected in to the abdominal cavity and the mice were sacrificed via the cervical dislocation method under the influence of the anesthetic. All animal experiments were approved by the Institutional Animal Care and Use Committee of Renji Hospital, School of Medicine, Shanghai Jiao Tong University (Shanghai, China). Aortas were harvested and cut into aortic rings of $\sim 1 \mathrm{~mm}$ thickness. A total of $1 / 2$ were immersed in DMEM with Cthrc1-expressing adenovirus $\left(2 \times 10^{7} \mathrm{pfu} / \mathrm{ml}\right)$, and the other $1 / 2$ were immersed in DMEM with the control adenovirus $\left(2 \times 10^{7} \mathrm{pfu} / \mathrm{ml}\right)$ at $37^{\circ} \mathrm{C}$. Following a total of $4 \mathrm{~h}$, the rings were placed in 96 -well culture plates and coated with liquid Matrigel ${ }^{\mathrm{TM}}$ (BD Biosciences, Franklin Lakes, NJ, USA). Following solidification of the Matrigel, $200 \mu 1$ Opti-MEM (Thermo Fisher Scientific, Inc.) with 2\% FBS was added. The aortic rings were incubated at $37^{\circ} \mathrm{C}$, and medium was changed every two days. Images of the aortic rings ( 3 fields per ring) were captured (original magnification, $\mathrm{x} 200$ ) on the eighth day with a microscope (Eclipse Ti-U; Nikon Corporation, Tokyo, Japan), the images were captured directly from the 96-well culture plates. Photoshop (version CS6; Adobe, San Jose, CA, USA) was used to sharpen the images and count the number of sprouting endothelia from the aortic ring for statistical analysis.

Cell proliferation assay. HUVECs were seeded in 96-well plates at a density of 2,000 cells/well and were incubated at $37^{\circ} \mathrm{C}$ overnight. Culture medium containing Cthrc1-expressing adenovirus or control adenovirus $\left(1 \times 10^{7} \mathrm{pfu} / \mathrm{ml}\right)$ was added into various wells, and the culture medium was changed after $2 \mathrm{~h}$. The optical density value of each group (six wells/group) was determined using the Cell Counting Kit-8 (CCK-8; Dojindo Molecular Technologies, Inc., Kumamoto, Japan) on days 0, 1, 2 and 3 , following the manufacturer's protocol.

Western blotting. Proteins were extracted from tissue samples using total protein extraction kits (cat. no. C510003; Sangon Biotech Co., Ltd., Shanghai, China). The protein concentration was determined using a BCA Protein Assay kit (cat. no. 23252; Thermo Fisher Scientific, Inc.). Proteins (40 $\mu \mathrm{g})$ were loaded and electrophoresed on a $10 \%$ SDS-PAGE gel. Proteins were transferred to a nitrocellulose membrane (EMD Millipore, Billerica, MA, USA), which was blocked using 5\% milk for $2 \mathrm{~h}$ at room temperature, and then incubated with the primary antibodies at $4^{\circ} \mathrm{C}$ overnight. An HRP-labeled secondary antibody (dilution, 1:5,000; cat. no. 7074; Cell Signaling Technology, Inc.) was added and incubated at room temperature for $1 \mathrm{~h}$. The immunoreactive signals were detected using Super Signal West Femto Maximum Sensitivity Substrate (Thermo Fisher Scientific, Inc.). The primary antibodies were anti-Cthrc1 (dilution, 1:1,000; cat. no. AP8778a; Abgent), anti-ERK1/2 (dilution, 1:1,000; cat. no. 9102; Cell Signaling Technology, Inc.), anti-phospho (P)-ERK1/2 (dilution, 1:1,000; cat. no. 4370; Cell Signaling Technology, Inc.), anti-JNK (dilution, 1:1,000; cat. no. 9252; Cell Signaling Technology, Inc.), anti-P-JNK (dilution, 1:1,000; cat. no. 4668; Cell Signaling Technology, Inc.) and anti- $\beta$-actin (dilution, 1:4,000; cat. no. 8457; Cell Signaling Technology, Inc.). Western blots were analyzed densitometrically using ImageJ software version $1.45 \mathrm{~S}$ (National Institutes of Health, Bethesda, MD, USA).

Tubule formation assay. Matrigel was pipetted into 48-well plates and allowed to polymerize for $30 \mathrm{~min}$ at $37^{\circ} \mathrm{C}$. HUVECs $\left(6 \times 10^{4}\right)$ were seeded on Matrigel in $200 \mu \mathrm{l}$ culture medium. The plates were incubated at $37^{\circ} \mathrm{C}$ for $6-8 \mathrm{~h}$, prior to the capture of images using an Eclipse Ti-U microscope (magnification, $\mathrm{x} 100$ ). ImageJ was used to measure the tube length in $\mu \mathrm{m}$. The sum of tube length was determined for statistical analysis.

Scratch wound assay. HUVECs were cultured to full confluence in 6-well culture plates. The cell layer was scratched using a sterile pipette tip and the scratched area was imaged 
A

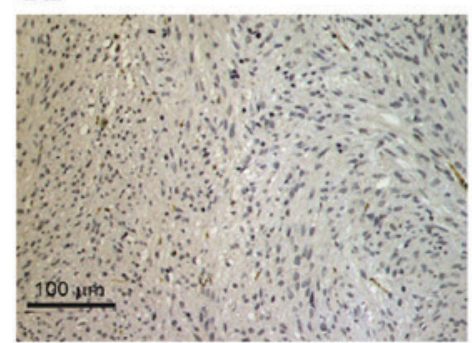

D

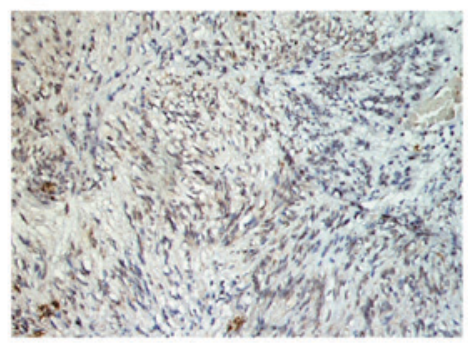

B

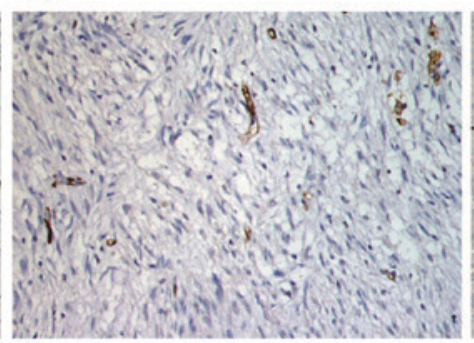

E

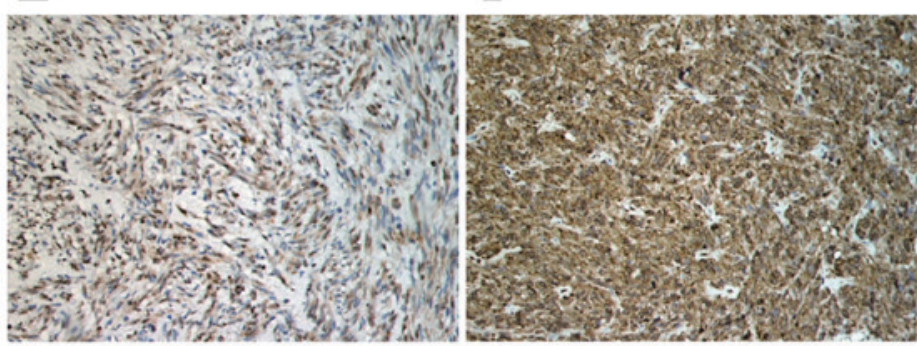

C

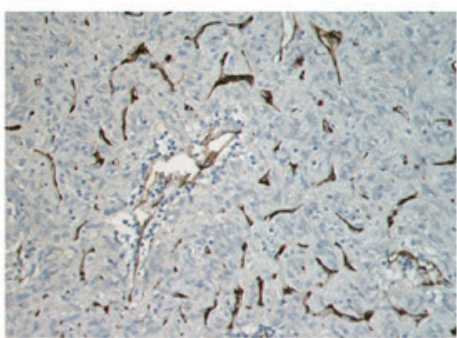

F

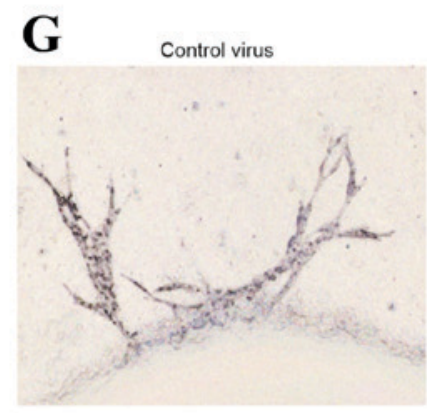

I

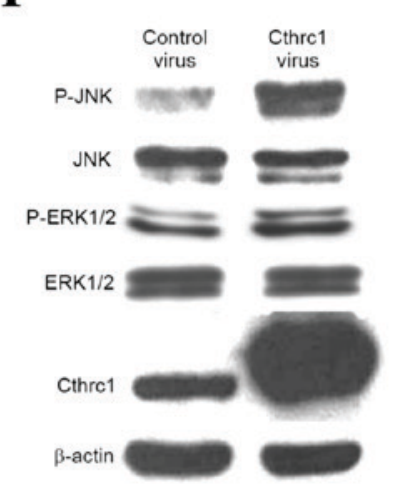

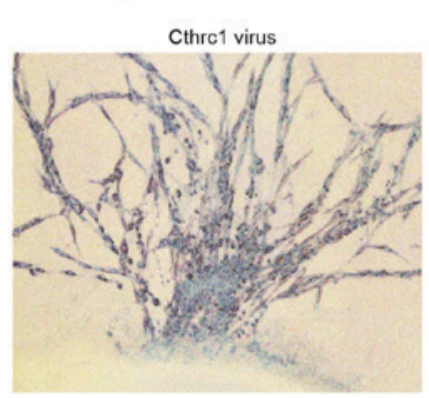

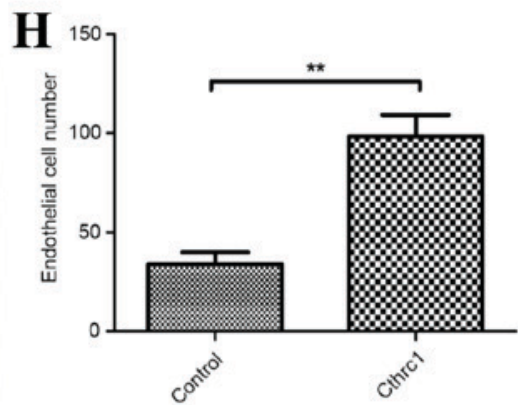

J

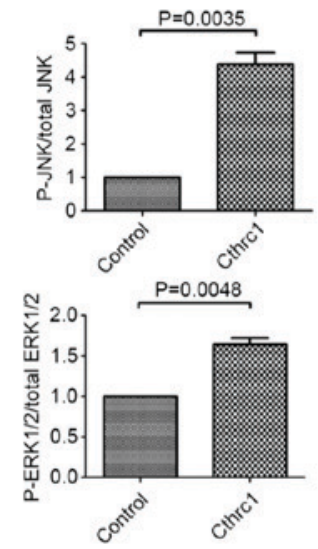

K

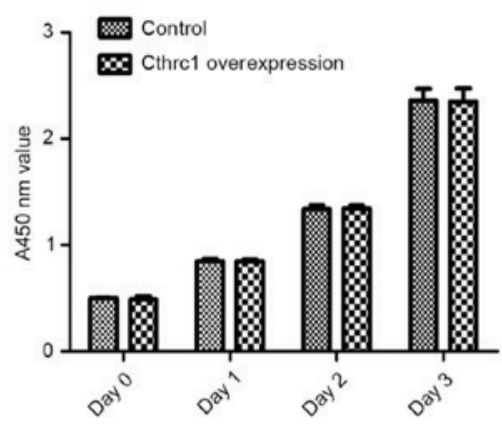

Figure 1. (A-C) IHC staining of different MVDs in gastrointestinal stromal tumor tissue arrays. Representative images of (D) +, (E) ++ and (F) +++ Cthrc1 IHC staining. (G) Expression of phosphorylated and total ERK1/2 and JNK in HUVECs treated with Con or Cthrc1-expressing adenovirus determined using western blotting. $\beta$-Actin was used as a loading control. (H) Quantitative analysis of the P-JNK/total JNK and P-ERK1/2/total ERK ratios determined from western blots. (I) Cell proliferation of HUVECs transfected with Con or Cthrc1-expressing adenovirus was determined using the Cell Counting Kit-8 assay. (J) Images of aortic rings treated with Con or Cthrc1-expressing adenovirus after 8 days. (K) Quantitative analysis of aortic ring-sprouting endothelial cells transfected with control or Cthrc1-expressing adenovirus after 8 days. ${ }^{* *} \mathrm{P}<0.001$. IHC, immunohistochemical; MVD, microvascular density; Cthrc1, collagen triple helix repeat containing 1; ERK, extracellular-signal-regulated kinase; JNK, c-Jun N-terminal kinase; HUVEC, human umbilical vein endothelial cell; P-, phospho.

with an Eclipse Ti-U microscope (original magnification, x200). Following incubation at $37^{\circ} \mathrm{C}$ for $24 \mathrm{~h}$, the scratched area was imaged again. ImageJ was used to measure the scratched area in $\mu \mathrm{m}^{2}$. The healing area was evaluated for statistical analysis.
Cell invasion assay. A Transwell chamber (24-well insert; pore size $8 \mu \mathrm{m}$; EMD Millipore) was coated with Matrigel for $6 \mathrm{~h}$ prior to the invasion assay. A total of $2 \times 10^{5}$ HUVECs were plated in the Transwell chamber with $200 \mu 1$ FBS-free DMEM. DMEM $(600 \mu \mathrm{l})$ containing 20\% FBS was added to the lower 
chambers of the 24-well plates. After $36 \mathrm{~h}$ of incubation at $37^{\circ} \mathrm{C}$, the cells that had invaded through the pores were fixed using $4 \%$ paraformaldehyde (25 $\mathrm{min}$ ) and stained using $0.5 \%$ crystal violet (30 $\mathrm{min}$ ) at room temperature, and the number of cells was counted under a x200 Eclipse Ti-U light microscope.

Statistical analysis. Each individual experiment was conducted with three or five replicates. All data are presented as the mean \pm standard error, and were analyzed using the Student's t-test or Mann-Whitney U test with SPSS (version 18.0; SPSS, Inc., Chicago, IL, USA). $\mathrm{P}<0.05$ was considered to indicate a statistically significant difference.

\section{Results}

MVD is associated with the tumor site and Cthrcl protein expression level. Quantification of the IHC staining demonstrated that there was a significant decrease in MVD in tumors located in the stomach compared with non-stomach-located tumors $(8.19 \pm 6.97$ vs. $16.48 \pm 17.04$, respectively; $\mathrm{P}=0.003$; Table I). GIST with increased expression of $\mathrm{Cthrc1}$ protein exhibited increased MVD compared with GIST with decreased expression of Cthrc1 (14.44 \pm 15.35 vs. $7.67 \pm 7.05$, respectively; $\mathrm{P}=0.021$; Table I). Statistical analysis indicated that MVD was not significantly associated with sex, mitotic index, tumor size or National Institutes of Health risk grade (Table I).

Effect of Cthrcl expression on aortic ring sprouting. To investigate the effect of Cthrcl on angiogenesis, an aortic ring assay was performed. The aortic ring assay demonstrated that Cthrcl-overexpressing aortic rings developed a significantly increased number of sprouting endothelial cells compared with the control $(\mathrm{P}<0.001)$, indicating that $\mathrm{C}$ thrc1 serves an important role in angiogenesis (Fig. $1 \mathrm{G}$ and $\mathrm{H}$ ).

Effect of Cthrcl overexpression on HUVEC proliferation, tubule formation, migration and invasion. Densitometric analysis of western blots (Fig. 1I) demonstrated significantly increased ratios of $\mathrm{P}-\mathrm{JNK} /$ total JNK $(\mathrm{P}=0.004)$ and $\mathrm{P}$-ERK1/2/total ERK1/2 $(\mathrm{P}=0.005)$ in HUVECs transfected with Cthrc1-expressing adenovirus compared with HUVECs transfected with control adenovirus (Fig. 1J). CCK-8 results demonstrated that HUVEC proliferation was not significantly affected by Cthrcl-expressing adenovirus (Fig. 1K), indicating that $\mathrm{Cthrc1}$ promotes aortic ring sprouting without promoting HUVEC proliferation. The scratch wound assay and the tubule formation assay revealed that $\mathrm{Cthrc1}$ protein overexpression was able to increase the migratory function of HUVECs (Fig. 2A and B), and increase HUVEC tubule formation (Fig. 3A and B). The cell invasion assay demonstrated that Cthrcl protein overexpression was able to increase HUVEC invasion (Fig. 3C and D). Decreased HUVEC migration and tubule formation were observed following treatment with the kinase inhibitors U0126 and SP600125 (Figs. 2A and B, $3 \mathrm{~A}$ and $\mathrm{B}$ ), suggesting that ERK1/2 and JNK phosphorylation may be the underlying molecular mechanism of promotion of angiogenesis by Cthrc1.

Effect of Cthrcl knockdown on HUVEC tubule formation, migration and invasion. Following Cthrc1 knockdown using
Table I. Characteristics of patients with gastrointestinal stromal tumors.

\begin{tabular}{|c|c|c|}
\hline Characteristic & $\begin{array}{l}\text { MVD (number } \\
\text { of vessels/field) }\end{array}$ & P-value \\
\hline Sex & & 0.899 \\
\hline Male $(n=33)$ & $12.45 \pm 13.70$ & \\
\hline Female $(n=27)$ & $13.11 \pm 14.62$ & \\
\hline Mitosis per $50 \mathrm{HPFs}$ & & 0.947 \\
\hline$<5(\mathrm{n}=32)$ & $12.91 \pm 13.68$ & \\
\hline$\geq 5(\mathrm{n}=28)$ & $12.57 \pm 14.62$ & \\
\hline Tumor size, $\mathrm{cm}$ & & 0.169 \\
\hline$<10(\mathrm{n}=44)$ & $11.57 \pm 12.13$ & \\
\hline$\geq 10(n=16)$ & $16.00 \pm 18.28$ & \\
\hline NIH risk grade & & 0.111 \\
\hline Low and intermediate $(n=41)$ & $11.73 \pm 12.50$ & \\
\hline High $(n=19)$ & $14.95 \pm 16.99$ & \\
\hline Tumor site & & $0.003^{\mathrm{a}}$ \\
\hline Stomach $(n=27)$ & $8.19 \pm 6.97$ & \\
\hline Non-stomach $(\mathrm{n}=33)$ & $16.48 \pm 17.04$ & \\
\hline Cthrc 1 protein expression & & $0.021^{\mathrm{a}}$ \\
\hline,$-+(n=15)$ & $7.67 \pm 7.05$ & \\
\hline,$+++++(n=45)$ & $14.44 \pm 15.35$ & \\
\hline
\end{tabular}

${ }^{\mathrm{a}} \mathrm{P}<0.05$. MVD, microvascular density; HPF, high-power field; NIH, National Institutes of Health; Cthrc1, collagen triple helix repeat containing 1; -, no tumor cells stained;,$+<25 \%$ tumor cells stained; ,$++ 25-50 \%$ tumor cells stained;,$+++>50 \%$ tumor cells stained.

siRNA in HUVECs, the ability of HUVECs to form tubules, migrate and invade was significantly decreased (Fig. 4), indicating that inhibition of Cthrc1 protein expression may be a promising method of inhibiting GIST angiogenesis.

\section{Discussion}

Previous studies have demonstrated that patients with increased expression of Cthrcl protein have a poorer prognosis compared with those with decreased expression, a phenomenon that has been observed in numerous types of tumor $(9-12,16)$. Tumor MVD is negatively associated with tumor prognosis. The present study demonstrated that Cthrc1 protein expression is associated with GIST MVD. The aortic ring assay demonstrated that Cthrc1 was able to promote aortic ring sprouting. The wound healing assay, cell invasion assay and tubule formation assay demonstrated that Cthrc1 was able to promote HUVEC migration, invasion and tubule formation. The results of the present study indicated that Cthrc1 serves an important role in GIST angiogenesis and may explain why patients with increased expression of Cthrc1 protein have a poorer prognosis.

Cthrc1 has been demonstrated to promote the phosphorylation of ERK and JNK in colon cancer (12) and GIST (9), respectively. Previous studies have revealed that the increase in phosphorylation of ERK and JNK may promote HUVEC 
A
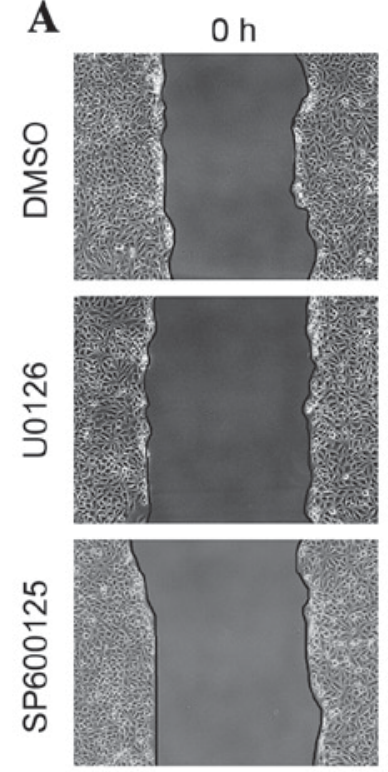

Control virus
$24 \mathrm{~h}$
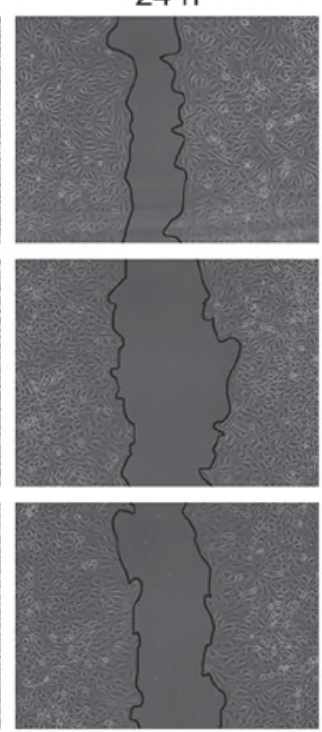
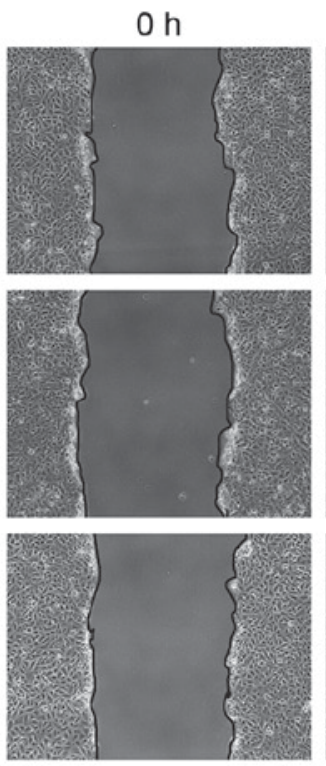

Cthrc1 virus

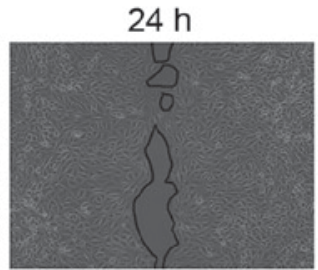

B

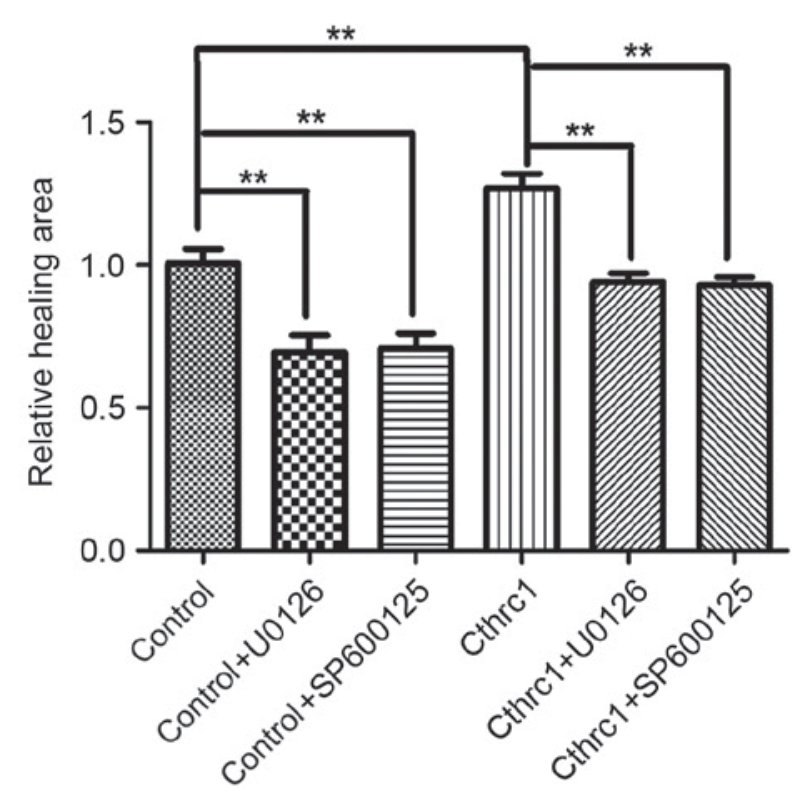

Figure 2. Effect of Cthrc1 overexpression on HUVEC migration. (A) Scratch wound assay of HUVECs treated with DMSO, U0126 and SP600125 following transfection with Con adenovirus and Cthrc1-expressing adenovirus. (B) Quantitative analysis of HUVEC wound healing. ${ }^{* *} \mathrm{P}<0.001$. HUVEC, human umbilical vein endothelial cell; DMSO, dimethyl sulfoxide; Cthrc1, collagen triple helix repeat containing 1.

migration and tubule formation (17-19). In the present study, it was demonstrated that Cthrc1 was able to promote ERK and JNK phosphorylation in HUVECs, and inhibition of ERK and JNK phosphorylation may decrease HUVEC migration and tubule formation. These results indicate that the pro-angiogenic effect of Cthrc1 is associated with the phosphorylation of ERK and JNK.

The planar cell polarity (PCP) signaling pathway is a highly conserved signaling cascade that coordinates epithelial and axonal morphogenic movements during organ development by regulating angiogenesis (20). Inhibition of the PCP signaling pathway disrupts endothelial cell growth, polarity and migration (20-22). Cthrc1 is a Wnt co-factor protein that selectively activates the Wnt/PCP signaling pathway by stabilizing ligand-receptor interactions $(9,23,24)$. Cthrc1 may activate the PCP signaling pathway; however, it was demonstrated to suppress the canonical Wnt signaling pathway in human embryonic kidney-293T and GIST cells $(9,23)$. Therefore, Cthrc1 may activate the PCP signaling pathway in HUVECs and promote tumor angiogenesis.

Collagen matrix deposition in ECM is an important process in the inhibition of tumor invasion and angiogenesis $(25,26)$. Cthrc1 may reduce collagen type I mRNA and protein levels in fibroblasts, resulting in decreased collagen synthesis and contributing to vascular remodeling (27). Matrix metalloproteinase 9 (MMP9) is an important factor in collagen degradation (28). Cthrc1 has been demonstrated to promote MMP9 secretion in colon cancer (12). Therefore, inhibiting Cthrcl may increase collagen matrix deposition and decrease tumor invasion and angiogenesis. 
A

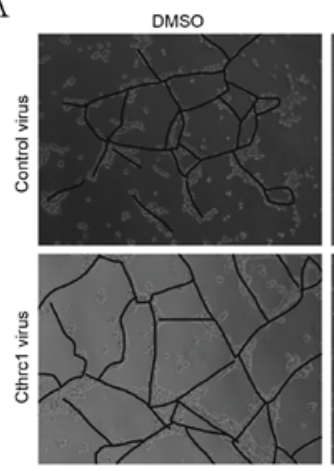

C

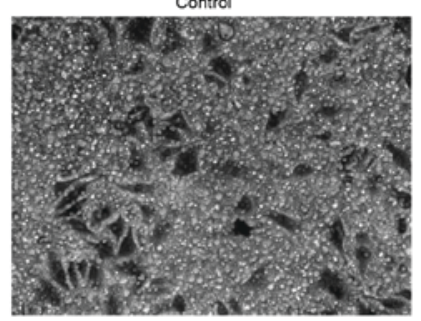

U0126
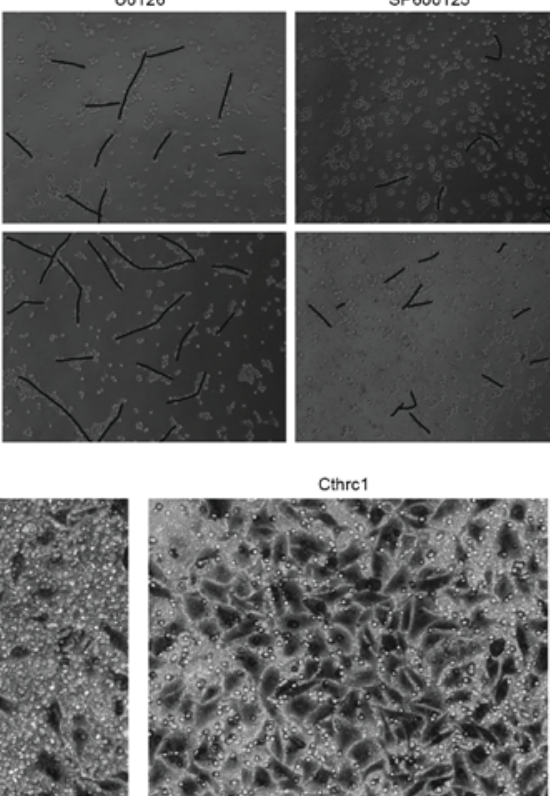

B

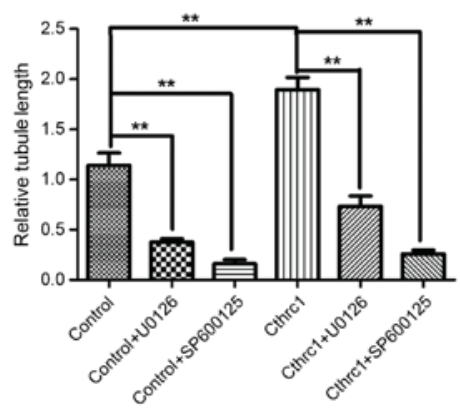

D

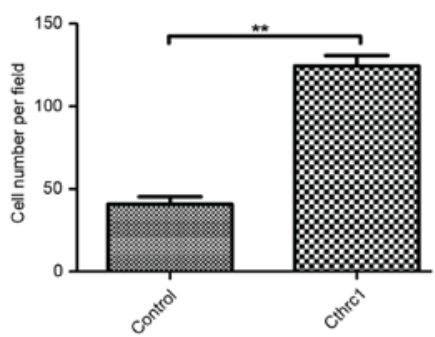

Figure 3. Effect of Cthrcl overexpression on HUVEC proliferation, tubule formation, migration and invasion. (A) Tubule formation assay of HUVECs treated with DMSO, U0126 and SP600125 in control and Cthrc1-expressing adenovirus groups. (B) Representative images of HUVECs transfected with control or Cthrc1-expressing adenovirus that had invaded through Matrigel to the bottom of the Transwell filter. (C) Quantitative analysis of tubule formation assay results. (D) Quantitative analysis of invasion assay results. ${ }^{* *} \mathrm{P}<0.001$. HUVEC, human umbilical vein endothelial cell; DMSO, dimethyl sulfoxide; Cthrc1, collagen triple helix repeat containing 1 .

A
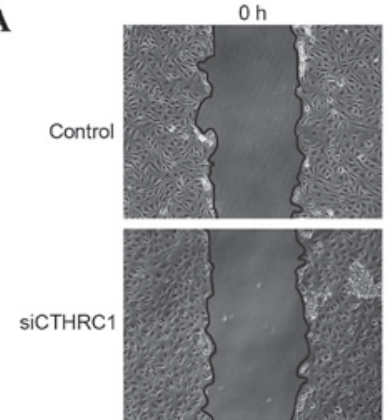

C

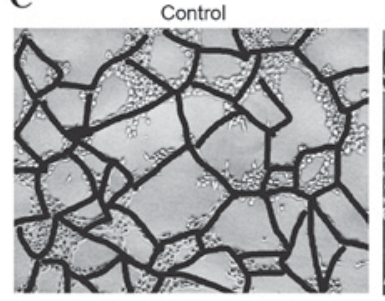

E

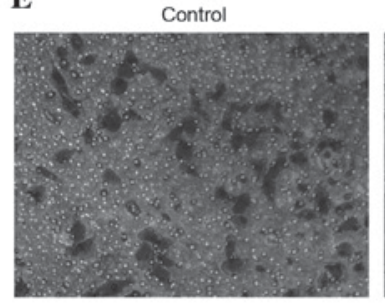

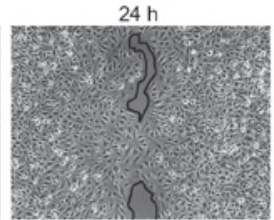

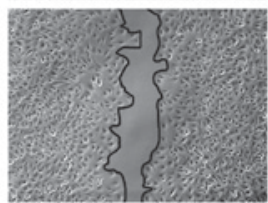

SiCTHRC1

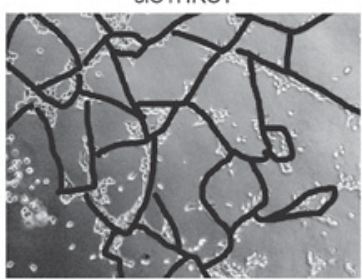

SICTHRC1

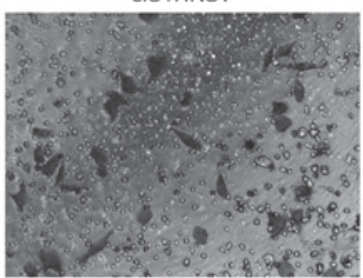

B

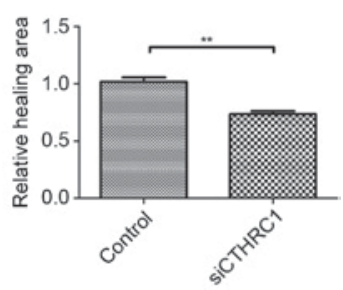

D

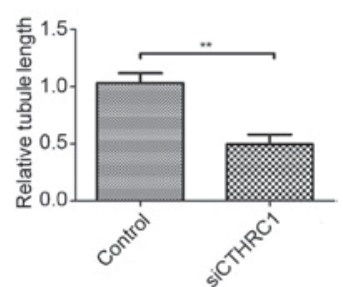

F

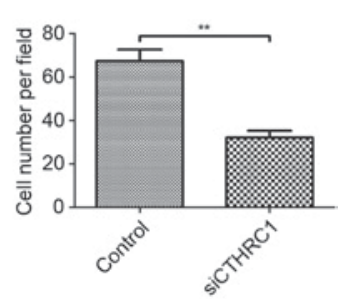

Figure 4. Cthrc1 knockdown decreases HUVEC tubule formation, migration and invasion. (A) Scratch wound assay of HUVECs treated with control siRNA or siCTHRC1. (B) Quantitative analysis of scratch wound assay results. (C) Tubule formation assay of HUVECs transfected with control siRNA or siCTHRC1. (D) Quantitative analysis of tubule formation assay results. (E) Cell invasion assay images of HUVECs transfected with control siRNA or siCTHRC1. (F) Quantitative analysis of invasion assay results. * $\mathrm{P}<0.001$. Cthrc1, collagen triple helix repeat containing 1; HUVEC, human umbilical vein endothelial cell; siRNA, small interfering RNA; siCTHRC1, siRNA targeting Cthrc1. 
Although the present study has revealed certain underlying molecular mechanisms regarding the pro-angiogenic effect of Cthrc1, further studies in vivo and in vitro are required to validate these experimental findings.

\section{Acknowledgements}

The present study was supported by the National Natural Science Foundation of China (grant no. 81270474). The authors of the present study thank Professor Xiong Ma (Shanghai Jiao-Tong University, Shanghai, China) for providing the Cthrc1-expressing adenovirus and the control adenovirus.

\section{References}

1. Carmeliet P and Jain RK: Angiogenesis in cancer and other diseases. Nature 407: 249-257, 2000.

2. Folkman J: Tumor angiogenesis: Therapeutic implications. N Eng J Med 285: 1182-1186, 1971.

3. Pollard JW: Tumour-educated macrophages promote tumour progression and metastasis. Nat Rev Cancer 4: 71-78, 2004.

4. Kalluri R and Zeisberg M: Fibroblasts in cancer. Nat Rev Cancer 6: 392-401, 2006.

5. Joyce JA and Pollard JW: Microenvironmental regulation of metastasis. Nat Rev Cancer 9: 239-252, 2009.

6. Lin WW and Karin M: A cytokine-mediated link between innate immunity, inflammation, and cancer. J Clin Invest 117: 1175-1183, 2007.

7. Pyagay P, Heroult M, Wang Q, Lehnert W, Belden J, Liaw L, Friesel RE and Lindner V: Collagen triple helix repeat containing 1, a novel secreted protein in injured and diseased arteries, inhibits collagen expression and promotes cell migration. Circ Res 96: 261-268, 2005.

8. Durmus T, LeClair RJ, Park KS, Terzic A, Yoon JK and Lindner V: Expression analysis of the novel gene collagen triple helix repeat containing-1 (Cthrc1). Gene Expr Patterns 6: 935-940, 2006.

9. Ma MZ, Zhuang C, Yang XM, Zhang ZZ, Ma H, Zhang WM, You H, Qin W, Gu J, Yang S, et al: CTHRC1 acts as a prognostic factor and promotes invasiveness of gastrointestinal stromal tumors by activating Wnt/PCP-Rho signaling. Neoplasia 16: 265-278, e1-13, 2014.

10. Hou M, Cheng Z, Shen H, He S, Li Y, Pan Y, Feng C, Chen X, Zhang Y, Lin M, et al: High expression of CTHRC1 promotes EMT of epithelial ovarian cancer (EOC) and is associated with poor prognosis. Oncotarget 6: 35813-35829, 2015.

11. Ke Z, He W, Lai Y, Guo X, Chen S, Li S, Wang Y and Wang L: Overexpression of collagen triple helix repeat containing 1 (CTHRC1) is associated with tumour aggressiveness and poor prognosis in human non-small cell lung cancer. Oncotarget 5: 9410-9424, 2014

12. Kim HC, Kim YS, Oh HW, Kim K, Oh SS, Kim JT, Kim BY, Lee SJ, Choe YK, Kim DH, et al: Collagen triple helix repeat containing 1 (CTHRC1) acts via ERK-dependent induction of MMP9 to promote invasion of colorectal cancer cells. Oncotarget 5: 519-529, 2014.

13. Wang P, Wang YC, Chen XY, Shen ZY, Cao H, Zhang YJ, Yu J, Zhu JD, Lu YY and Fang JY: CTHRC1 is upregulated by promoter demethylation and transforming growth factor- $\beta 1$ and may be associated with metastasis in human gastric cancer. Cancer Sci 103: 1327-1333, 2012.
14. Liu X, Liu B, Cui Y, Wang F, Sun H and Lv F: Collagen triple helix repeat containing 1 (Cthrc1) is an independently prognostic biomarker of non-small cell lung cancers with cigarette smoke. Tumour Biol 35: 11677-11683, 2014.

15. Bian Z, Miao Q, Zhong W, Zhang H, Wang Q, Peng Y, Chen X, Guo C, Shen L, Yang F, et al: Treatment of cholestatic fibrosis by altering gene expression of Cthrc1: Implications for autoimmune and non-autoimmune liver disease. J Autoimmun 63: 76-87, 2015.

16. Gu L, Liu L, Zhong L, Bai Y, Sui H, Wei X, Zhang W, Huang P, Gao D, Kong Y and Lou G: Cthrcl overexpression is an independent prognostic marker in gastric cancer. Hum Pathol 45: 1031-1038, 2014.

17. Wu F, Song H, Zhang Y, Zhang Y, Mu Q, Jiang M, Wang F, Zhang $\mathrm{W}, \mathrm{Li} \mathrm{L}, \mathrm{Li} \mathrm{H}$, et al: Irisin induces angiogenesis in human umbilical vein endothelial cells in vitro and in Zebrafish Embryos in vivo via activation of the ERK signaling pathway. PLoS One 10: e0134662, 2015.

18. Lee OH, Kim YM, Lee YM, Moon EJ, Lee DJ, Kim JH, Kim KW and Kwon YG: Sphingosine 1-phosphate induces angiogenesis: Its angiogenic action and signaling mechanism in human umbilical vein endothelial cells. Biochem Biophys Res Commun 264: 743-750, 1999.

19. Jin YJ, Park I, Hong IK, Byun HJ, Choi J, Kim YM and Lee H: Fibronectin and vitronectin induce AP-1-mediated matrix metalloproteinase-9 expression through integrin $\alpha(5) \beta(1) / \alpha(v)$ $\beta(3)$-dependent Akt, ERK and JNK signaling pathways in human umbilical vein endothelial cells. Cell Signal 23: 125-134, 2011.

20. Cirone P, Lin S, Griesbach HL, Zhang Y, Slusarski DC and Crews CM: A role for planar cell polarity signaling in angiogenesis. Angiogenesis 11: 347-360, 2008.

21. Ju R, Cirone P, Lin S, Griesbach H, Slusarski DC and Crews CM: Activation of the planar cell polarity formin DAAM1 leads to inhibition of endothelial cell proliferation, migration, and angiogenesis. Proc Natl Acad Sci USA 107: 6906-6911, 2010.

22. Descamps B, Sewduth R, Ferreira Tojais N, Jaspard B, Reynaud A, Sohet F, Lacolley P, Allières C, Lamazière JM, Moreau C, et al: Frizzled 4 regulates arterial network organization through noncanonical Wnt/planar cell polarity signaling. Circ Res 110: 47-58, 2012.

23. Yamamoto S, Nishimura O, Misaki K, Nishita M, Minami Y, Yonemura S, Tarui $\mathrm{H}$ and Sasaki H: Cthrcl selectively activates the planar cell polarity pathway of Wnt signaling by stabilizing the Wnt-receptor complex. Dev Cell 15: 23-36, 2008.

24. Kelley MW: Leading Wnt down a PCP path: Cthrc1 acts as a coreceptor in the Wnt-PCP pathway. Dev Cell 15: 7-8, 2008.

25. Chang $\mathrm{C}$ and Werb Z: The many faces of metalloproteases: Cell growth, invasion, angiogenesis and metastasis. Trends Cell Biol 11: S37-S43, 2001.

26. Cox G and O'Byrne KJ: Matrix metalloproteinases and cancer. Anticancer Res 21: 4207-4219, 2001

27. LeClair R and Lindner V: The role of collagen triple helix repeat containing 1 in injured arteries, collagen expression, and transforming growth factor beta signaling. Trends Cardiovasc Med 17: 202-205, 2007.

28. Burg-Roderfeld M, Roderfeld M, Wagner S, Henkel C, Grötzinger J and Roeb E: MMP-9-hemopexin domain hampers adhesion and migration of colorectal cancer cells. Int J Oncol 30: 985-992, 2007. 